\title{
Initial Antinoise Performance Analysis of Pupil Phase Diversity Based on Genetic Algorithm
}

\author{
Huizhen Yang and Yaoqiu Li \\ School of Electronic Engineering, Huaihai Institute of Technology, Lianyungang 222005, China \\ Correspondence should be addressed to Huizhen Yang; yangandxu@yeah.net
}

Received 27 April 2013; Revised 3 July 2013; Accepted 30 July 2013

Academic Editor: Anthony Kenyon

Copyright ( $) 2013$ H. Yang and Y. Li. This is an open access article distributed under the Creative Commons Attribution License, which permits unrestricted use, distribution, and reproduction in any medium, provided the original work is properly cited.

\begin{abstract}
Pupil phase diversity (PPD) wavefront sensor is a new kind of phase-visualization methods, and the output signal of PPD represents the input pupil phase and shows a 1-1 mapping between the position of the wavefront error in the pupil and its position in the output signal. High-precisely wavefront measuring can be obtained under no noise by using appropriate phase restoration algorithm while performance of PPD under noise is unknown. We analyzed antinoise performance of PPD based on genetic algorithm (GA) through measuring the distorted wavefront under different noise level. Simulation results show that wavefront measuring is almost not affected by the existence of noise, which indicates that PPD based on GA can be used in applications with noise.
\end{abstract}

\section{Introduction}

Phase diversity (PD) refers to a method of image-based wavefront sensing where multiple images of an unknown extended object or scene are used to estimate both the unknown phase parameters and the unknown object $[1,2]$. A quadratically distorted diffraction grating can be used to simultaneously image multiple object planes onto a single detector [3]. The diffraction grating provides a different level of defocus in each diffraction order, and the intensity images formed on a CCD detector provide data for the $\mathrm{PD}$ algorithm, which is called as defocus PD (DPD). Campbell et al. put forward the generalized phase diversity (GPD) method in 2004 [4]. The GPD, like the DPD sensor, uses two intensity images to perform wavefront sensing and has more extensive applications. However, DPD's use of two images that are symmetrically defocused with respect to the unknown wavefront is replaced in GPD by a pair of images of the wavefront plane, each convolved with arbitrary but related aberration functions. These functions may include, but not be limited to, defocus. GPD has two different kinds of optics constructions, one is based on image plane and the other based on pupil plane [5].

In this paper, we use the optics constructions of pupil plane and call them pupil phase diversity (PPD). High-precisely wavefront measuring can be obtained under no noise by using appropriate phase restoration algorithm while performance of PPD wavefront sensor under noise is unknown. In this paper, we analyze the performance of PPD sensor in confronting noise through measuring the distorted wavefront under different noise level. The most widely used algorithms for phase retrieval of PD wavefront sensor are GerchbergSaxton approach [6] or its modified editions and iteration algorithms [7]. Previous approaches can not take full advantage of specialty of PPD wavefront sensor, which represents the input pupil phase and shows a 1-1 mapping between the position of the wavefront error in the pupil and its position in the difference of the intensity images. We decompose the output signal by using Zernike polynomial and then use genetic algorithm (GA) $[8,9]$ to search the optimum solution. Based on simulation mode, the performance of PPD wavefront sensor under noise is investigated.

\section{The Principle of Pupil Phase Diversity}

Figure 1 gives the optical construction of PPD wavefront sensor, where $\phi(r)$ is the input distorted wavefront $(r=\{x, y\}$ is a vector in the plane orthogonal to the optical axes) and $D r$ is the output information of PPD wavefront sensor.

The output information of PPD wavefront sensor, $D r$, is defined as the difference of a pair of the intensity images 


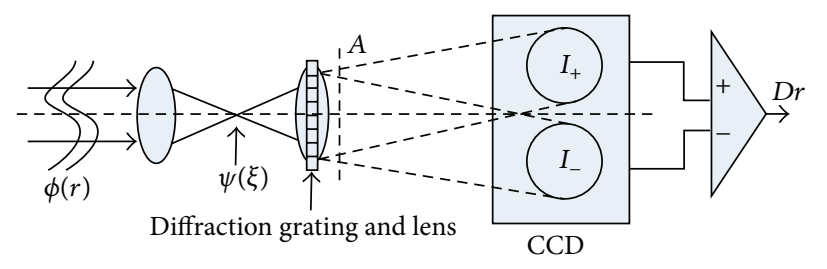

FIGURE 1: Schematic of the PPD wavefront sensor.

formed in the \pm 1 diffraction orders. These intensity images are themselves created by the convolution of the input wavefront with the filter function programmed into a diffraction grating. From Figure 1, Dr could be expressed as

$$
\begin{aligned}
D r= & I_{+}-I_{-}=\left|\mathfrak{I}\left(\mathfrak{I}\left(e^{i \phi(r)}\right) * e^{i \phi_{d+}}\right)\right|^{2} \\
& -\left|\mathfrak{I}\left(\mathfrak{I}\left(e^{i \phi(r)}\right) * e^{i \phi_{d-}}\right)\right|^{2} \\
= & \left|e^{i \phi(r)} \otimes \mathfrak{J}\left(e^{i \phi_{d+}}\right)\right|^{2}-\left|e^{i \phi(r)} \otimes \mathfrak{J}\left(e^{i \phi_{d-}}\right)\right|^{2},
\end{aligned}
$$

where $\mathfrak{I}$ is the operation of Fourier Transform, $\otimes$ is the operation of convolution, and $e^{i \phi_{d}}$ is the function applied by the grating ( $\phi_{d}$ is the diversity phase). Note that the output information of PPD wavefront sensor shows details of the input wavefront by convolution operation.

\section{Phase Retrieval of PPD Wavefront Sensing Genetic Algorithm}

The PPD uses two intensity images to perform wavefront sensing, and its output signal $D r$ represents the relative location of the error of wavefront aberration. We try to decompose the output signal $\mathrm{Dr}$ of the sensor by using the Zernike polynomial firstly and then make use of GA to find the optimum solution, so that the phase restoration algorithm can converge both rapidly and precisely. The phase restoration algorithm can be divided into two steps: decomposition and optimization. The results of the decomposition are used as the initial solution of the optimization. Decomposition step can speed the search process, and optimization step can obtain high precision of phase restoration. The flow chart of phase retrieval based on mode decomposition and GA is shown in Figure 2.

We use the combinations of Zernike functions to describe the distorted wavefront $\phi(r)$ :

$$
\phi(r)=\sum_{j=1}^{N} \alpha_{j} Z_{j}(r)
$$

where $N$ is the number of terms in the parameterization and $Z_{j}(r)$ is the $j$ th member of the basis set. The vector $\left\{\alpha_{j}\right\}$ is the parameter to be searched by phase retrieval algorithm.

From the basic principle of PPD wavefront sensor, we know that the output signal $\mathrm{Dr}$ represents the relative location of the error of wavefront aberration. So, when the input wavefront is a plane wavefront, that is, there is no aberration, the output signal $\mathrm{Dr}$ should be a plane. With the evolution

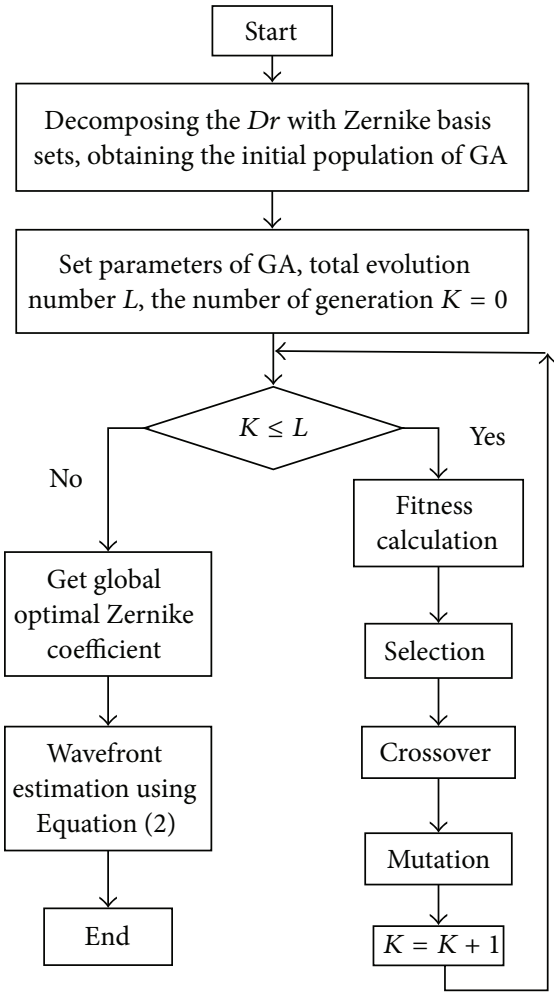

Figure 2: The flow chart of phase retrieval based on mode decomposition and GA.

of population, the current solution of $\left\{\alpha_{j}\right\}$ approaches the optimum solution gradually, and the current $D r_{\text {curr }}$ also approaches the initial $D r_{\text {init }}$. The fitness function is defined as follows:

$$
J=\sum_{(x, y)}\left(D r_{\text {init }}(x, y)-D r_{\text {curr }}(x, y)\right)^{2}
$$

The closer to the optimum solution the current $\left\{\alpha_{j}\right\}$, the smaller the fitness function $J$.

\section{Results and Analysis}

We programme the wavefront sensing method in Matlab environment according to the simulation model and perform the evolution process over the phase screen to be measured. The method proposed by N. Roddier, which makes use of a Zernike expansion of randomly weighted KarhunenLoeve functions, is used to simulate atmospherically distorted 


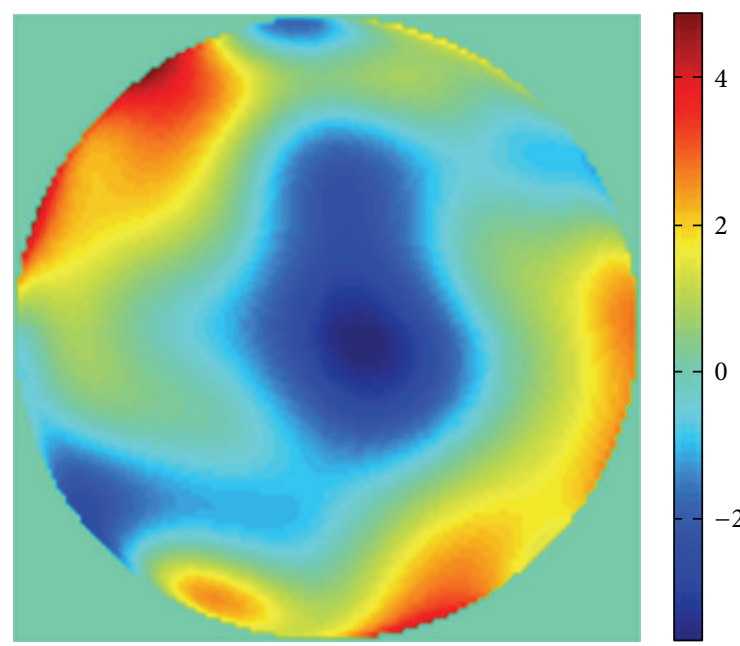

(a)

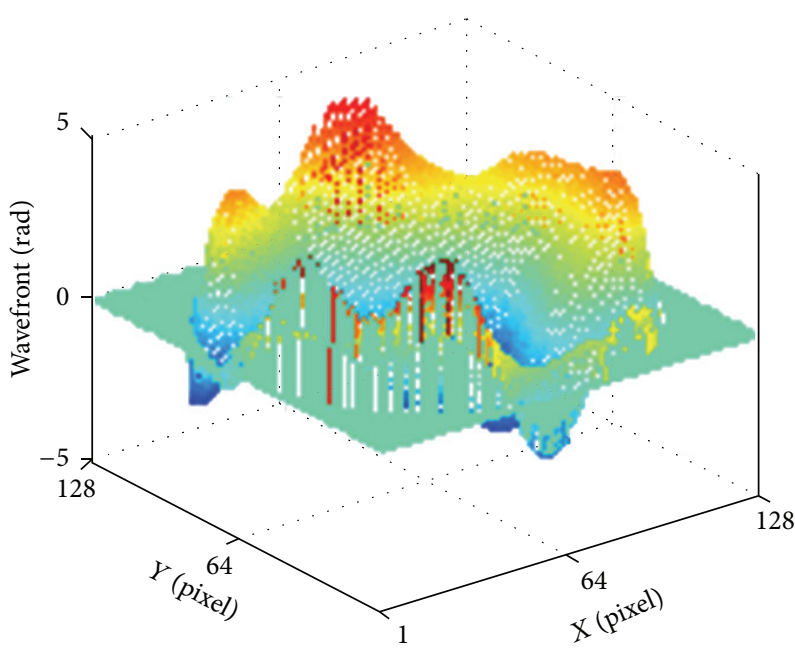

(c)

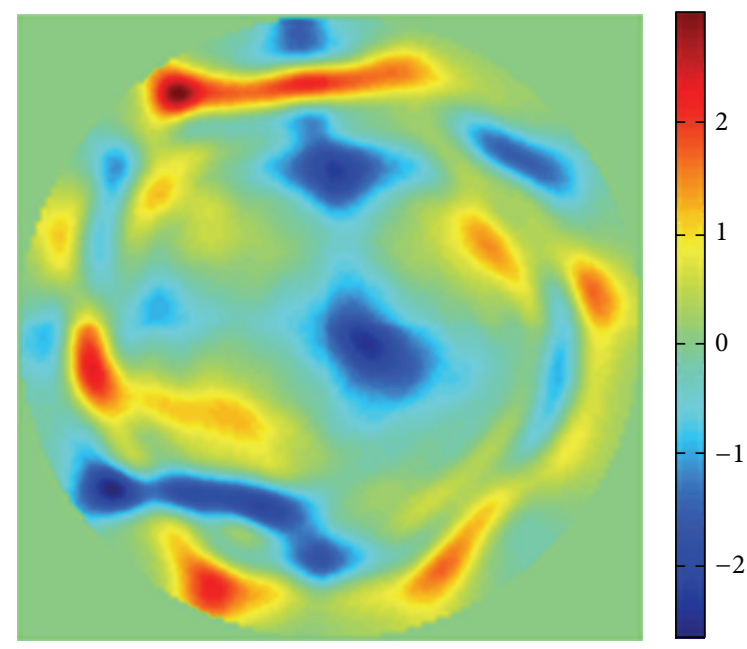

(b)

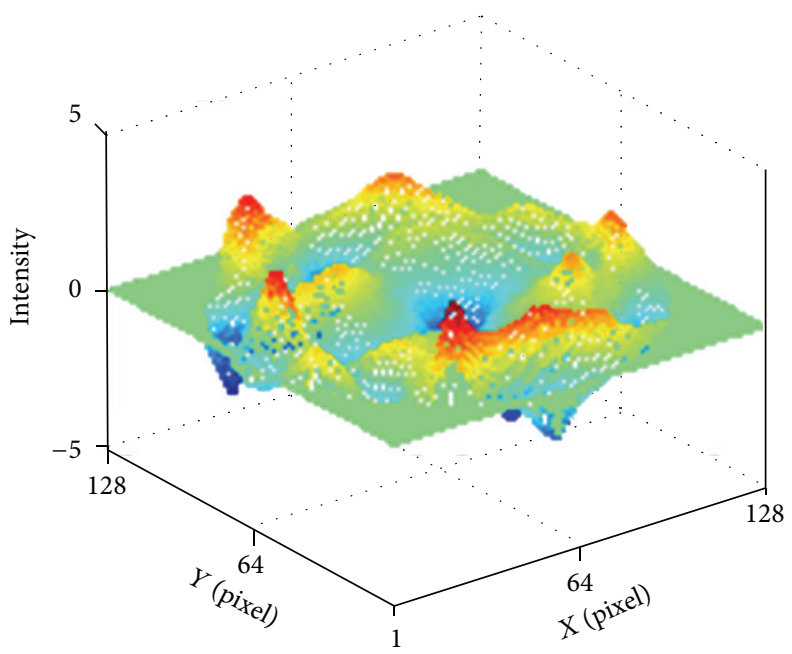

(d)

FIgURE 3: The wavefront to be measured (a) and (c) (scale in rad) and the output signals, Dr, of PPD wavefront sensor with a defocus aberration filter function (b) and (d) (scale in contrast).

wavefronts [10]. Considering that the low-order aberrations (tilts, defocus, astigmatism, etc.) have the most significant impact on optics system quality, we use the first 65 Zernike polynomial orders. Different phase screens generated according to this method are not correlated to each other and represent the Kolmogorov spectrum. The phase screens $\phi(r)$ are defined over $128 \times 128$ pixels and do not include the tip/tilt aberrations. The tip/tilt aberrations are usually controlled by another control algorithm and are considered as being removed completely in our simulation.

The output signal of the PPD wavefront sensor, $D r$, is generated by the difference of a pair of intensity images formed in the \pm diffraction order. The two intensity images suffer the same noise type because they are sampled from the same CCD. Noise meets the additive Gaussian noise model in high-level photon or CCD readout noise-based circumstances. We first add the Gaussian noise to the pair of intensity images, respectively, and then calculate the difference of the two intensity images. The equation SNR $\left.=10 \log _{10}\left(\sum(\operatorname{Dr}(x, y))^{2}\right) / \sum(N(x, y))^{2}\right)$ (SNR: signalnoise raito) is used to estimate the scale of noise, where $N(x, y)$ is noise of Gaussian distribution.

The root of mean square (RMS) of distorted wavefront to be restored is 1.56 , rad and peak and valley (PV) value is $8.45 \mathrm{rad}$. To estimate correction capability, we also use another metric, Strehl ratio (SR), which is defined as the ratio of the maximum intensities of the distorted point spread function and the diffraction-limited point spread function. In optical application the SR has a value between 0 and 1 where an SR of 1 would be a perfect wavefront. The initial SR value of distorted wavefront to be measured is 0.14 . The defocus aberration with a PV value of 1 wavelength is used as the filter function. The wavefront to be measured is given in Figure 3 . Figures 3(a) and 3(c) show two-dimensional image and threedimensional images of distorted wavefront, respectively, and Figures 3(b) and 3(d) are those of output signals of PPD 
TABLE 1: Comparison of the original wavefront and the residual wavefront in RMS, PV, and SR.

\begin{tabular}{|c|c|c|c|c|c|}
\hline & \multirow{2}{*}{ Original wavefront } & \multicolumn{4}{|c|}{ Residual wavefront } \\
\hline & & No noise & $\mathrm{SNR}=30 \mathrm{~dB}$ & $\mathrm{SNR}=20 \mathrm{~dB}$ & $\mathrm{SNR}=10 \mathrm{~dB}$ \\
\hline $\mathrm{PV}$ (rad) & 8.45 & 0.25 & 0.31 & 0.44 & 0.46 \\
\hline RMS (rad) & 1.56 & 0.05 & 0.052 & 0.053 & 0.052 \\
\hline SR & 0.14 & 0.998 & 0.998 & 0.997 & 0.997 \\
\hline
\end{tabular}

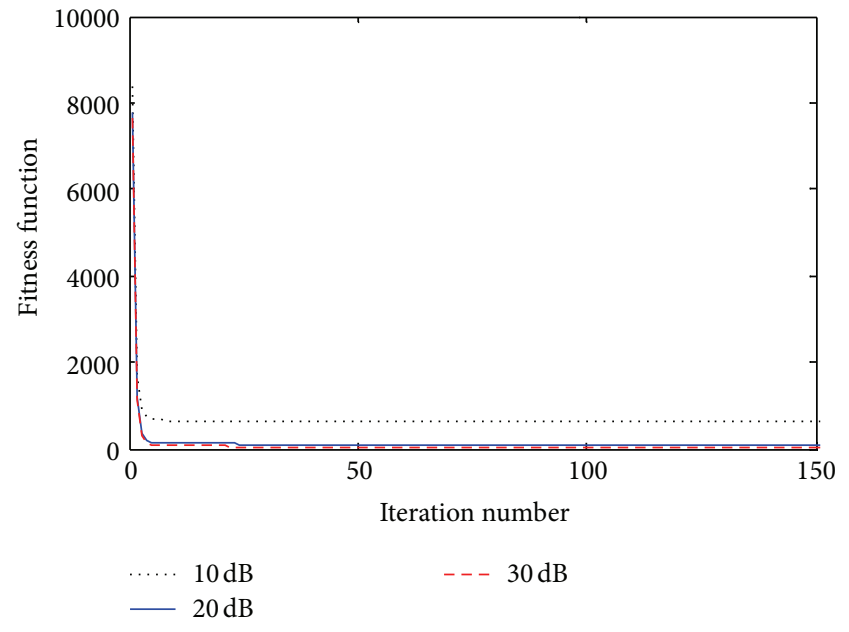

FIGURE 4: Iteration curves of the fitness function during decomposition step and GA evolution under different noise level.

wavefront sensor. Comparing Figure 3(a) with Figure 3(b) and Figure 3(c) with Figure 3(d), we can get that the location of the wavefront error can be found from the output signals of the PPD wavefront sensor.

Figure 4 gives evolution curves of the metric function $J$ with the adaptation process when SNR is $10 \mathrm{~dB}, 20 \mathrm{~dB}$, and $30 \mathrm{~dB}$ respectively. Three evolution curves all can converge rapidly after decomposition step, and GA optimization further offers the accuracy of wavefront measuring. The stronger the noise, the bigger the initial fitness function, and the fitness function under SNR = 10 is the biggest in three scales of noise after the end of evolution.

The residual wavefront, which is not measured successfully, can be calculated through the difference between the original wavefront and the measured wavefront. Values of RMS, PV, and SR are listed in Table 1. Compared with data of the original wavefront, RMS and PV of the residual wavefront under three scales of noise decrease greatly, and SR increases from 0.14 to 0.997 or 0.998 , which approximate to the ideal value 1 . Noticeably, the performance of wavefront measuring is almost not affected by the existence of noise.

Zernike coefficient comparison between the distorted wavefront to be measured and the restoration wavefront under several scales of noise is given in Figure 5. Considering the resolution of figure, we only show 3-10 order coefficient. From Figure 5, we can see that the measured phases under several scales of noise are nearly identical to the original.

Initial output signal of PPD wavefront sensor and residual wavefront under three scales of noise are shown in Figure 6.

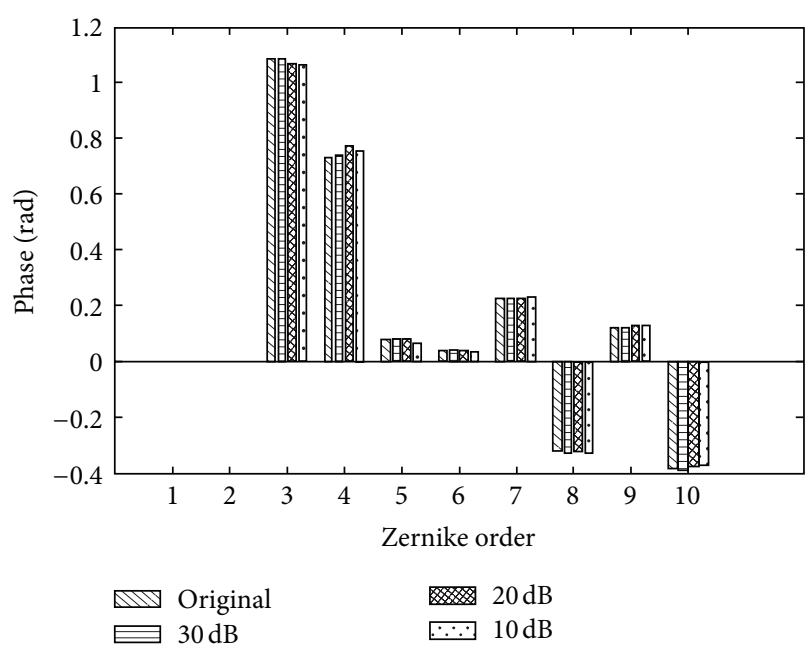

FIGURE 5: Zernike coefficient comparison.

SNR of images is $30 \mathrm{~dB}, 20 \mathrm{~dB}$, and $10 \mathrm{~dB}$, respectively, from left column to right column, and initial output signal of PPD is in the upper row and residual wavefront in the lower row. Compared with the original wavefront in Figure 3, residual wavefronts in Figure 6 are very small, which indicates that the original phase can be measured successfully. Although the residual wavefront increases as the noise scale increases, values of SR in Table 1 are all near to ideal value. Above results show that the method we offer can high-precisely measure the phase from the output signal of the PPD wavefront sensor, and the performance of wavefront measuring is almost not affected by the existence of noise.

\section{Conclusion}

GPD wavefront sensor extends the classical PD algorithm and has more extensive applications. The PPD wavefront sensor, one kind of GPD sensor, makes use of the optics construction based on pupil plane. The output signal of PPD sensor represents the input pupil phase and shows a 1-1 mapping between the position of the wavefront error in the pupil and its position in the difference of the intensity images. High-precisely wavefront measuring can be obtained under no noise by using appropriate phase restoration algorithm while performance of PPD wavefront sensor under noise is unknown. We analyze the performance of PPD sensor confronting noise through measuring the distorted wavefront under different noise level. Simulation results show that the performance of wavefront measuring is almost not affected by the existence 

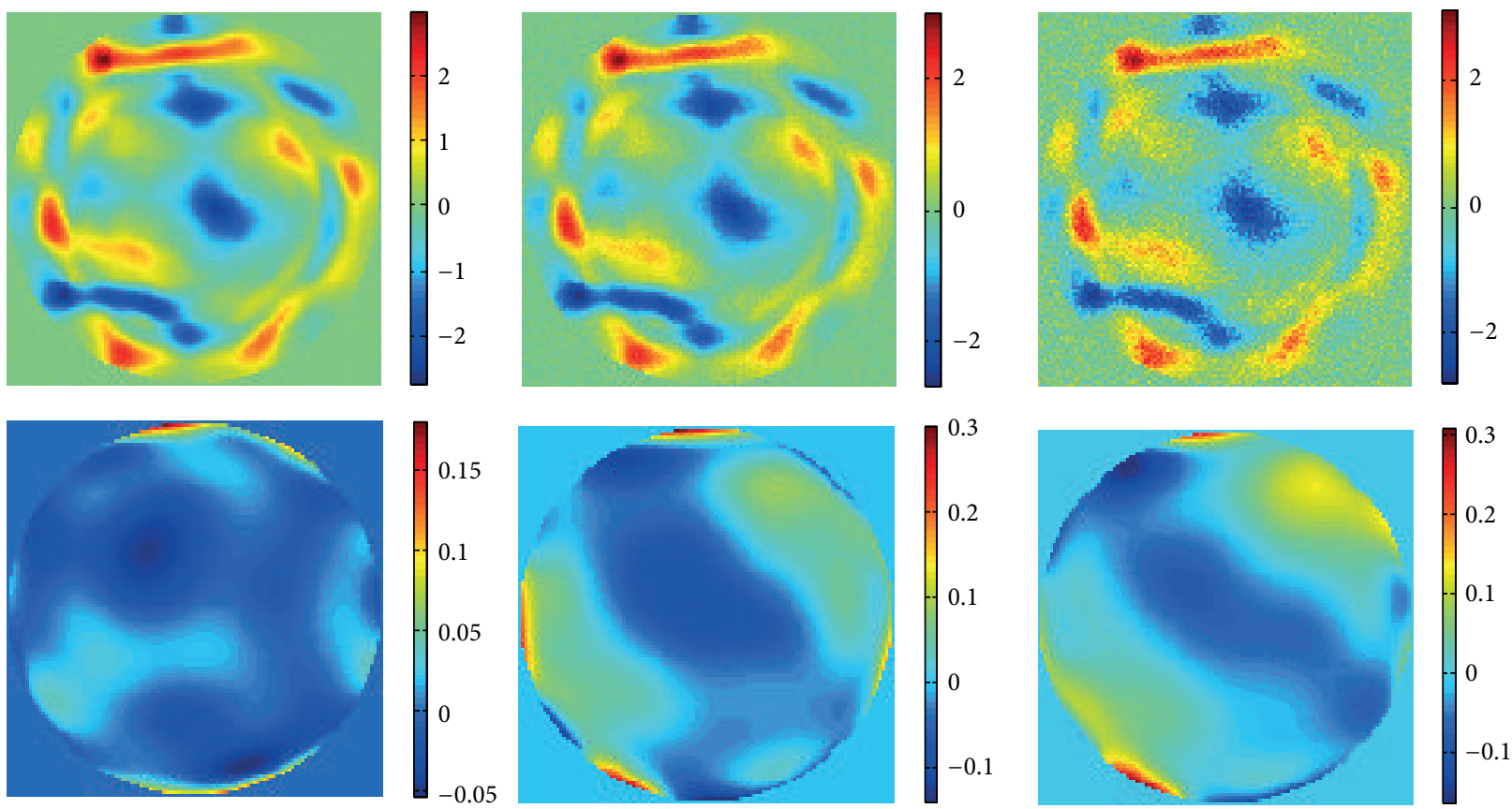

FIGURE 6: Initial output signal of PPD wavefront sensor and residual wavefront under three scales of noise. SNR of images is $30 \mathrm{~dB}, 20 \mathrm{~dB}$, and $10 \mathrm{~dB}$, respectively, from left column to right column. Initial outputs are in the upper row, and residual wavefronts are in the lower row.

of noise, which indicates that PPD wavefront sensor can be used in applications under noise and offer a new measuring method for wavefront sensing with noise.

\section{Acknowledgments}

This work is supported by Natural Science Fund for Colleges and Universities in Jiangsu Province under Grant no. 11KJB510001 and by the Priority Academic Program Development of Jiangsu Higher Education Institutions.

\section{References}

[1] R. G. Paxman, T. J. Schulz, and J. R. Fienup, "Joint estimation of object and aberrations by using phase diversity," The Journal of the Optical Society of America A, vol. 9, no. 7, pp. 1072-1085, 1992.

[2] M. R. Bolcar and J. R. Fienup, "Sub-aperture piston phase diversity for segmented and multi-aperture systems," Applied Optics, vol. 48, no. 1, pp. A5-A12, 2009.

[3] P. M. Blanchard and A. H. Greenaway, "Simultaneous multiplane imaging with a distorted diffraction grating," Applied Optics, vol. 38, no. 32, pp. 6692-6699, 1999.

[4] H. I. Campbell, S. Zhang, A. H. Greenaway, and S. Restaino, "Generalized phase diversity for wave-front sensing," Optics Letters, vol. 29, no. 23, pp. 2707-2709, 2004.

[5] H. I. Campbell, Generalized phase diversity wavefront sensing [Doctoral thesis], Heriot-Watt University, 2006.

[6] R. W. Gerchberg and W. O. Saxton, "A practical algorithm for the determination of phase from image and diffraction plane pictures," Optik, vol. 35, no. 2, pp. 237-250, 1972.

[7] J. R. Fienup, "Phase retrieval algorithms: a comparison," Applied Optics, vol. 21, no. 15, pp. 2758-2769, 1982.
[8] J. H. Holland, "Genetic algorithms and the optimal allocation of trials," SIAM Journal on Computing, vol. 2, no. 2, pp. 88-105, 1973.

[9] H. Yang and Y. Li, "Genetic algorithm for phase retrieval of generalized phase diversity," in Proceedings of the International Conference on Energy Systems and Electrical Power (ESEP '11), vol. 13, pp. 4806-4811, December 2011.

[10] N. Roddier, "Atmospheric wavefront simulation using Zernike polynomials," Optical Engineering, vol. 29, no. 10, pp. 1174-1180, 1990. 

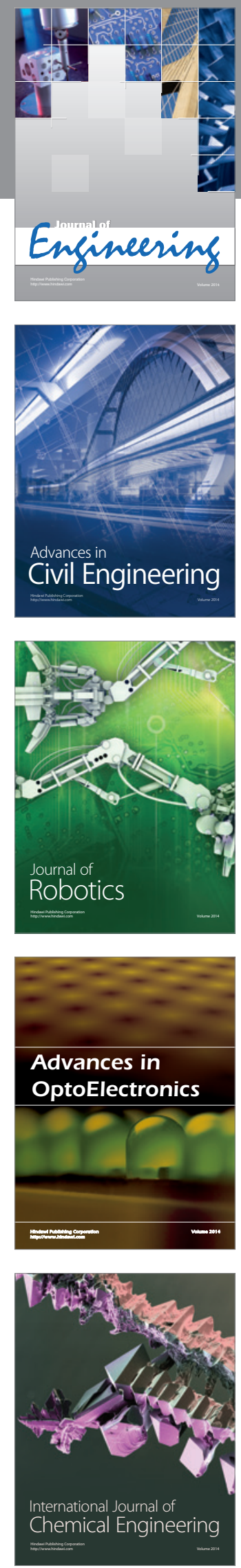

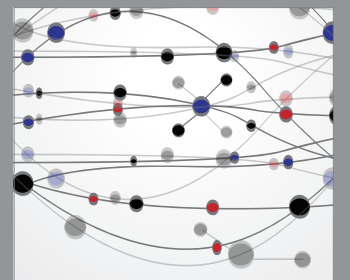

The Scientific World Journal
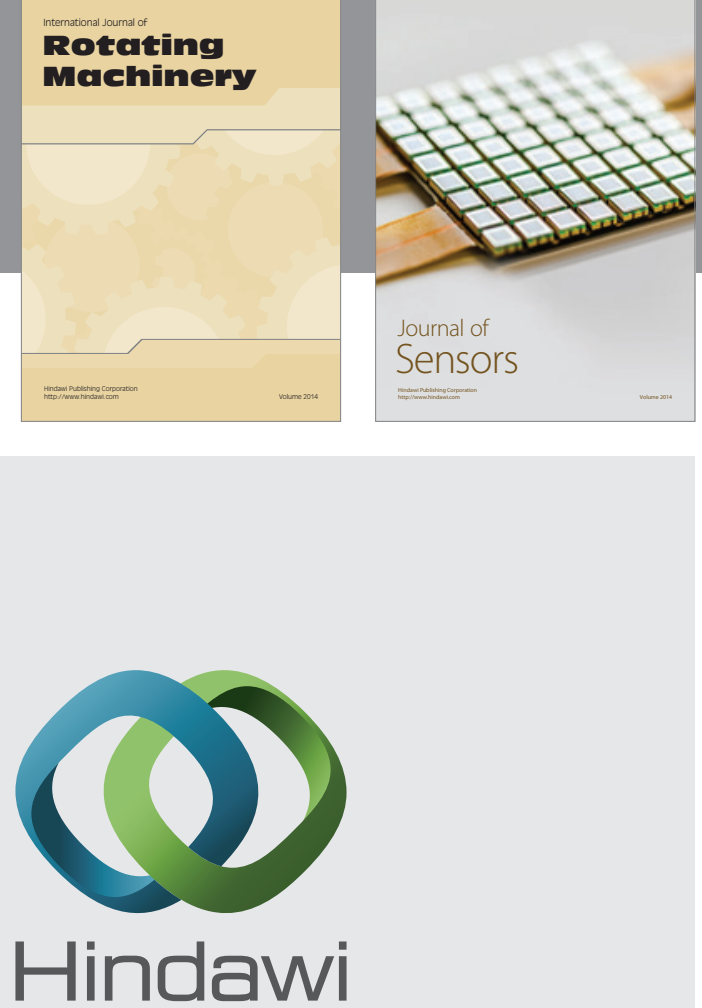

Submit your manuscripts at http://www.hindawi.com
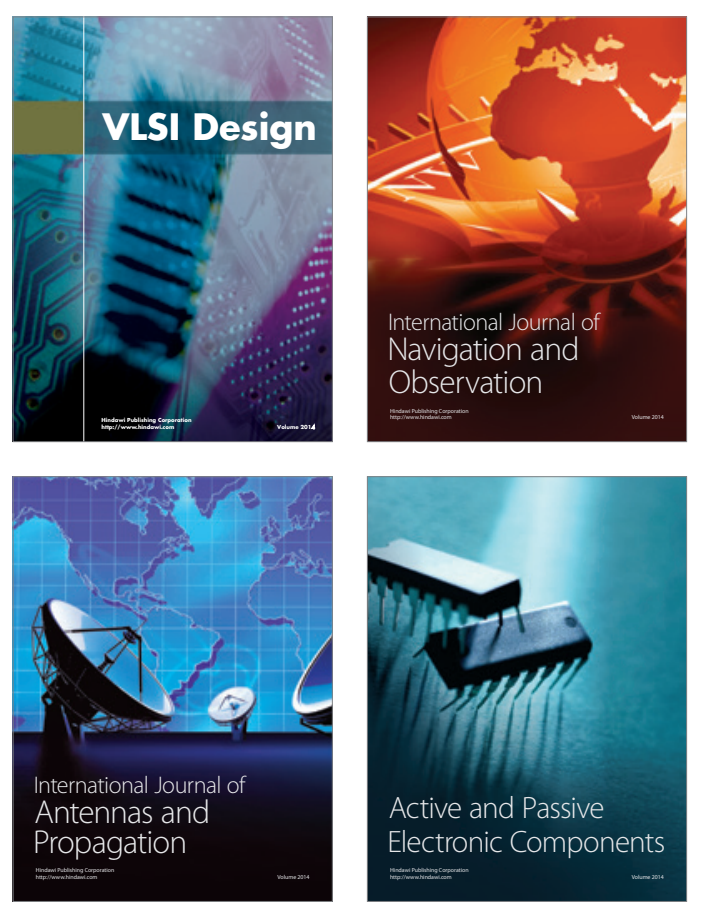
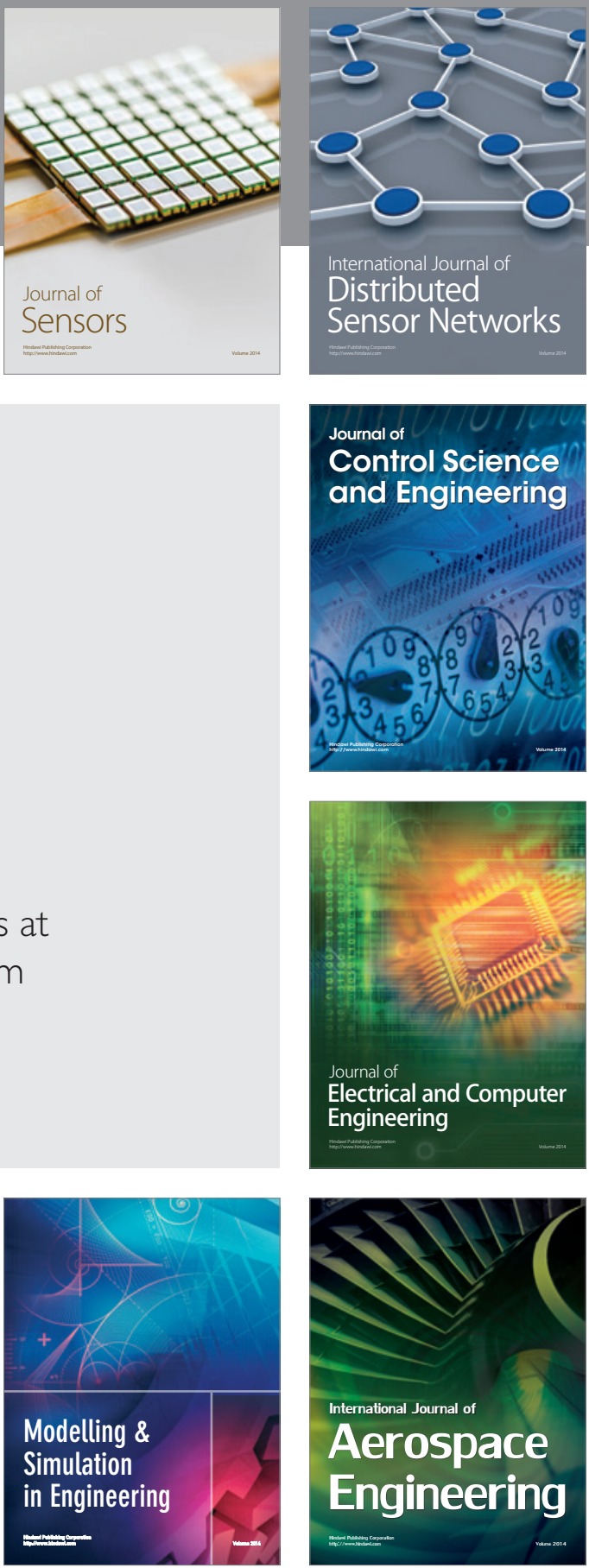

Journal of

Control Science

and Engineering
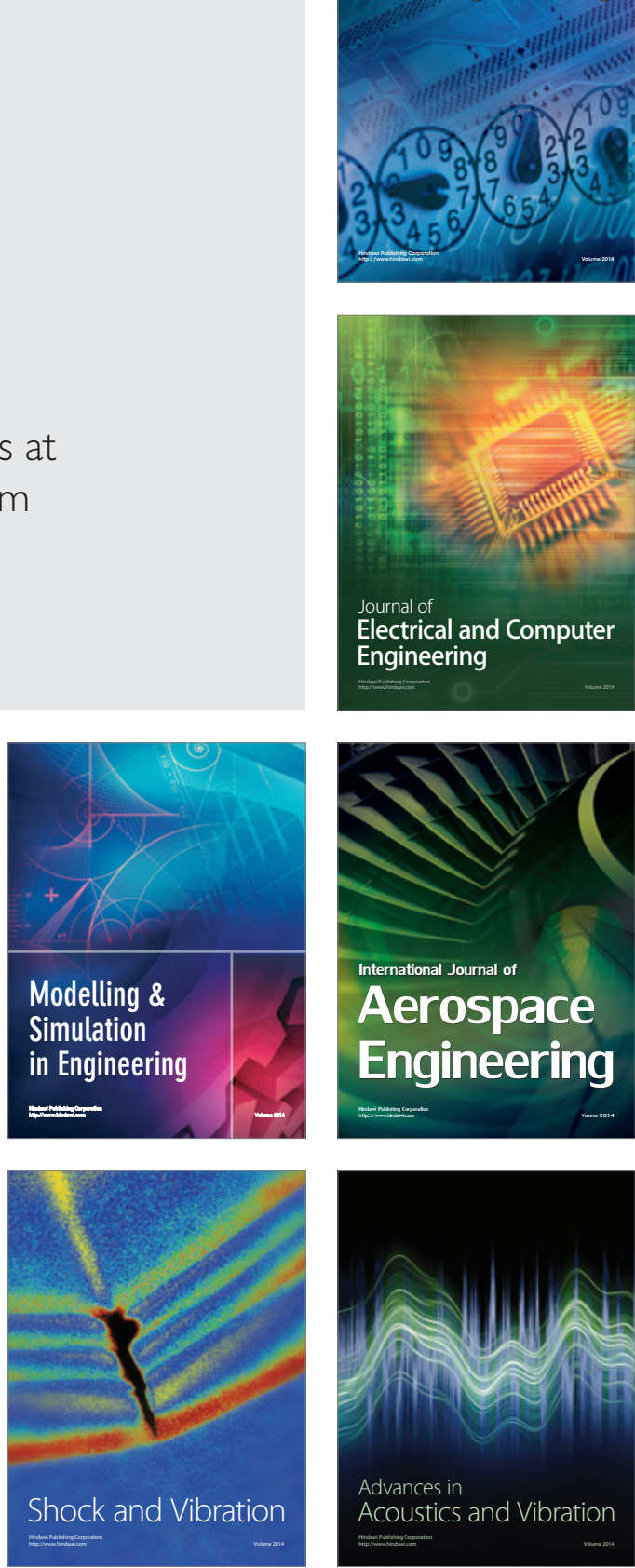